\title{
Critical Review of the Evolving Essence of the Science of Nursing: Complexity Integration Nursing Theory (CINT) E-Book
}

\section{Frances Zaragoza ${ }^{1}$ and Catherine Bailey ${ }^{2^{*}}$}

${ }^{1}$ Graduate Nursing Program, Texas Woman's University, USA

${ }^{2}$ Associate Professor, The Houston J. and Florence A. Doswell College of Nursing, Texas Woman's University, USA

\begin{abstract}
The following paper is a critical review of the e-book The Evolving Essence of the Science of Nursing: The Complexity Integration Nursing Theory (CINT). The CINT provides an opportunity for nurses and advanced practice nurses to gain insight into themselves and integrate nursing knowledge into practice. The critical book review analyzed the CINT thesis, development of the CINT theory, theoretical underpinning, contributions to practice, significance to academia, logic, and readability, as well as bias, influences, strengths, and weaknesses. A comparison made between the CINT and Jarrin's (2007) integral philosophy and definition of nursing in the context of the advance practice nurse. The CINT offers a multifaceted, yet valuable understanding of nursing for nurses of all levels.
\end{abstract}

\section{Publication History:}

Received: January 27, 2017

Accepted: April 03, 2017

Published: April 05, 2017

\section{Keywords:}

Advanced practice nursing, Complexity integration nursing theory, Critical book review, Nursing theory

\section{Introduction}

Nursing professors and theorists Dr. Sharon L. Van Sell and Ioannis A. Kalofissudis's 169-page book discussed complexity integration nursing theory (CINT), a metatheory comprised of four grand nursing theories: (a) the theory of nursing knowledge, (b) the theory of nursing practice, (c) the human being nursing theory, and (d) the social entirety nursing theory [1].ICU Web Journalpublished the book in Athens, Greece, and the book republished as a second printing in 2010. This book review conducted by Frances Zaragoza, RN, BS, MS, and Catherine Bailey, RN, BS, MS, Ph.D., CNE, considered the following topics: (a) thesis statement of CINT; (b) contributions to scholarly community and nursing practice; (c) book's logic, readability, research, and theoretical foundations; (d) theory similar to CINT; (e) book's biases; (f) book's strengths and weaknesses; (g) book's theoretical underpinnings; and (h)application to advanced practice nursing and research.

\section{Thesis Statement of CINT}

The theory of nursing practice formula stated, "The depth and breadth of practice $(\mathrm{P})$ is determined by the individual nurse's (IB) integration and synthesis through cognitive, affective, psychomotor, and spiritual domains of self, times nursing knowledge (NK)" [1].This grand theory served as the thesis of CINT and the theory's theoretical basis. The grand theory of nursing knowledge (NK) listed four fulcrums: (a) nursing foundation, defined as knowledge of science and humanities; (b) methodology, a problem-solving process supporting self-observation methodology; (c) nursing essence, comprised of components of the nursing profession including acknowledgement of all nursing theories past, present, and future; and (d) disciplined inquiry, defined as research. Theoretical bases supporting the individual nurse (IB) were the theory of and future nursing theories. A major construct of CINT was Plato's Allegory of the Cave which was a metaphor that showed how deceptive nursing practice, which encompassed the human being nursing theory and the social entity nursing theory. The theoretical underpinnings were important because the individual nurse is a human being. "The human being based on fundamental concepts of The Being, Philosophy, Science and civilization incorporated in Culture with continuous energy flow to sustain life and drive life cycles involving observation, development, application and evolution" [1, P. 58]. In addition, "the fundamental concepts are interconnected; sustains the human being as a complex open living system with the HOLON (whole or entirely)" [1, P.58]. "The HYPER-HOLON, space outside of the HOLON circle, represents the transcended individual who as a human intellect of the world and simultaneously as a separate observer of the world can perceive the linking for the existence of the HYPER-HOLON" [1, P.58]. Also, as a participant "the social entity is both a network within the HOLON and a miniature network of the HOLON organization. Humanity is a network within the Social Entirety network. The Social Entirety is not a measurable structure, but a pattern of networks" [1, P. 62].The four grand nursing theories, nursing knowledge, nursing practice, human being, and social entirety, integrate forming a complex nursing metatheory.

The thesis statement declaratively conveyed the main points Van Sell and Kalofissudis [1]were trying to make. Although the scope of the thesis statement was broad, the thesis was refined, and each point raised was thoroughly explored in the content of the book. The only weakness of the thesis statement was with the multiple elements involved, which can be confusing at times and required multiple readings to understand the CINT's concepts. Despite this shortcoming, the thesis statement effectively represented the entire theory and presented an arguable claim.

\section{Contributions and Significance to Academia, the Scholarly Community, or Best Practices}

Nurses must be socialized into the nursing profession to respond to the needs of the rapidly changing healthcare environment. Education

"Corresponding Author: Dr. Catherine Bailey, The Houston J. and Florence A Doswell College of Nursing, 5500 Southwestern Medical Avenue, \#7211, Dallas, Texas, USA, 75235-7299. Tel: 1-214-689-6571, FAX: 214-689-6539; E-mail: cbailey2@twu.edu

Citation: Zaragoza F, Bailey C (2017) Critical Review of the Evolving Essence of the Science of Nursing: Complexity Integration Nursing Theory (CINT) E-Book. Int J Nurs Clin Pract 4: 228. doi: https://doi.org/10.15344/2394-4978/2017/228

Copyright: (c) 2017 Zaragoza et al. This is an open-access article distributed under the terms of the Creative Commons Attribution License, which permits unrestricted use, distribution, and reproduction in any medium, provided the original author and source are credited. 
played a big role in socializing the individual to understand the language of the nursing profession. CINT was significant to academia, the scholarly community, and best practices by defining "agents of socialization within the nursing profession" [1]. CINT also formed a "comprehensive umbrella for multiple practice applications" [1]. For example, because CINT accountedfor the past, present, and future nursing theories, CINT can be applied not only to present practice but also to evolving and predictable patterns in nursing practice and research.

\section{Logic, Readability, and Research}

Van Sell and Kalofissudis [1] showed the logic of the content in the e-book through the theory formulas, which provided a structure of the concepts and elements. The chapters went into depth about the theory andCINT's foundations, and the e-book presented support to the theory's claims. The readability of the prose, however, was deemed to be difficult due to complex vocabulary and concepts throughout the book. For example, someone who has had no prior knowledge of philosophy or physics will find difficulty in understanding the metaphor of Plato's Allegory of the Cave as well as the reference to the principle of entropy. Because of these complex concepts, the reader may experience difficulty in linking how exactly these concepts all apply to nursing. Despite these challenges, the book was wellresearched, and the content was adequately supported, evidenced by an extensive reference list and availability of citations to claims offered in the book. The footnotes throughout the book helped with understanding the concepts presented.

Albert Einstein and Florence Nightingale greatly influenced Van Sell and Kalofissudis [1]during the formation of CINT. Nightingale's philosophy of human beings in the center of a social framework resonated throughout CINT. Einstein, on the other hand, was the foundation for and origin of the concept of nursing as science and art.

\section{Comparison With Theories Similar to CINT}

Van Sell and Kalofissudis [1] determined CINT is a metatheory, which is the highest level of theory. A similar metatheory to CINT is Olga Jarrin's [2-4] integral theory. The purpose of the integral theory was to have a "unifying meta-theory, which retains all the diversity of nursing while providing common ground for communication" within the nursing profession (para. 1). One of the foundations of the integral theory was called quadrants, which are self and consciousness, brain and organism, culture and worldview, and social system and environment [2]. The integral theory utilized the method of synchrony, which is similar to CINT self-observation, to provide a framework for asking questions.Furthermore, the topic of nursing as science and artand the human being as central to social and ecological webs were important to the integral theory. Additionally, Jarrin adopted her theory from Ken Wilber's integral psychology [5] and following soon after was Dorsey's theory of integral nursing [6] derived from Ken Wilber's extensive work with integral theories.

Contrasting to Jarrin's [2]integral theory, CINT had a more philosophical base and was more developed regarding its concepts [1]. Another difference between CINT and theintegral theory was the focus of each theory. In CINT, the human being, especially the individual nurse (IB), was the focus, while caring was Jarrin's integral theory's main focus.

\section{Biases or Influences}

Although nurse educators tested and supported the grand theory of nursing knowledge and practice at the Greenville Technical College, the book by Van Sell and Kalofissudis [1]did not show any further studies regarding the rest of the grand theories that comprise CINT. From the book alone, then, the readerwas unable to assume that the rest of the theories were tested, which led to questioning if CINT metatheory applies to nursing practice as a whole or just the grand theories alone. Van Sell and Kalofissudisstated that a "true metatheory in nursing will emerge as a superstructure with multiple practical applications and extensive opportunities for [research]" [1]. The prior statement maybe considered biased towards approval of CINT since no additional references or experiments were cited in the book to support the statement. However, since the publishing of the CINT, nurse researchers have successfully applied CINT as a nursing research framework [7-8].

\section{Strengths and Weaknesses}

A merit and strength of CINTwerethe theory's ability to be applied to all areas of nursing-staff nursing, advanced practice nursing, nursing administration, nursing education, and nursing research. CINT had an extensive range that did not limit its scope for application. The individual nurse was encouraged to draw on all or part of other nursing theories to facilitate the best outcome. Another strength of CINT was the provision of situational examples. These examples truly helped the reader understand the concepts of the theory and how CINT can be applicable in nursing practice. Finally, twoadditional major strengths of CINT werethe theory's theoretical and philosophical foundation along with the research and support provided to sustain the theory.

The main shortcoming and weakness of CINT were the difficulty of the printed material. The e-book by Van Sell and Kalofissudis [1]contained complex concepts with multiple broad and abstract components, making the material hard to read at times. A reader needed to have an optimal level of expertise and knowledge to understand and relate to the theory, although the examples helped with understanding some concepts. Putting the explanations in simpler terms would have been helpful and would have made the book more reader-friendly and inviting for nurses to read.

\section{Theoretical Underpinnings}

The readers observed three theoretical underpinnings in CINTthe deep ecology framework, the human being as the center of the entirety, and nursing as science and art [1]. Deep ecology was the fundamental interconnectedness of all phenomena, and the basis for the theory's concept that everything in society is interconnected to one another and developing the connection between the patient and the environment can yield beneficial results. Second, the human being as the center of the entirety was recognized throughout CINT, especially the human being theory and the social entirety nursing theory. The human being was considered a complex, living system interconnected with philosophy, science, and culture, and the individual nurse (IB) was recognized as a human being.

Einstein proposed that experiences portrayed in logicwould be considered a science. However, experiences recognized intuitively would be considered art.Therefore, Van Sell and Kalofissudis [1] demonstrated Einstein's proposal for the nursing profession thus resulting in recognition that nursing is both science and art. 
Citation: Zaragoza F, Bailey C (2017) Critical Review of the Evolving Essence of the Science of Nursing: Complexity Integration Nursing Theory (CINT) E-Book. Int J Nurs Clin Pract 4: 228. doi: https://doi.org/10.15344/2394-4978/2017/228

Page 3 of 3

Furthermore, Florence Nightingale also suggested that nursing is an art and requires just as much devotion and hard work as any painter's or sculptor's work.Clearly, nursing is both science and art because nursing incorporates logic, assessment, and evaluation with intuition and reflection. For example, nurses get an intuitive feeling if their patient's condition is declining, independent of data such as vital signs and laboratory values.

\section{CINT and Advanced Practice Nursing and Nursing Research}

Advanced practice nursing and nursing research will be able to apply CINT through the theory of nursing practice and nursing knowledge formula to identify which factors nurses can modify to improve their practice [1]. An advanced practice nurse (APN) will be able to realize that not only scientific knowledge is necessary to provide good care, but knowledge about policies, research, and nursing theories is essential.In addition, APNs can use self-observation as a tool to organize each nurse's thinking. Through self-observation,the APN will be able to integrate and synthesize the multiple domains of self with nursing knowledge. For example, an APN can reflect not only on the patient's immediate needs but also on needs often overlooked like environment and sensations.

CINT is a nursing theory about nursing theories; therefore, APNs and nursing research can use CINT to predict trends in nursing, socialize nurses into the profession, and free society from being trapped in the Platonic cave. Nurses Van Sell and Kalofissudis [1] developed CINT for other nurses to move the profession forward. The CINT offers a true unifying practical metatheory for the profession of nursing.

\section{Competing Interests}

The authors declare that they have no competing interests.

\section{Funding}

This article was published with support from Texas Woman's University Libraries' Open Access Fund.

\section{References}

1. Van Sell SL, Kalofissudis IA (2002) The evolving essence of the science of nursing: Complexity integration nursing theory [E-book]. Athens, Greece: ICU Web Journal.

2. Jarrin OF (2007) An integral philosophy and definition of nursing. School of Nursing Scholarly Works 2: 4.

3. Jarrin OF (2007) An integral philosophy and definition of nursing. J Integral Theory Pract 2: 79-100.

4. Jarrin OF (2012) The integrality of situated caring in nursing and the environment. Advances in Nursing Science 35: 14-24.

5. Wilber K (2002) Integral Psychology. Boston: Shambhala.

6. Dossey BM (2008) Theory of integral nursing. Advances in Nursing Science 31: E52-E73.

7. Binner M, Ross D, \& Browner I (2011) Chemotherapy-Induced Peripheral Neuropathy: Assessment of Oncology Nurses' Knowledge and Practice. Oncol Nurs Forum 38: 448-454.

8. Jeffery AD, Steffen-Mutsch K, Knapp L (2014) Knowledge and Recognition of SIRS and Sepsis among Pediatric Nurses. Pediatric Nursing 40: 271278 . 